\title{
Study on the Preparation Technology for High Pure Gallium Nitrate
}

\author{
Quanmao Yü \\ Institute of Functional Materials of Jiangxi University of Finance \& Economics, Nanchang 330013, \\ P.R. China;
}

Keywords: Gallium nitrate; Preparation technology; High pressure - low pressure condensation method

\begin{abstract}
The highly efficient High Pressure - Condensation process is designed and studied to synthesis high purity Gallium nitrate. The luminescent properties of phosphor materials using raw materials produced by this progress are investigated. The results show that Gallium nitrate can obtain by following process conditions: The molar ratio of the law materials Gallium oxide and nitric acid is 1:8 (nitrate concentration is $8 \mathrm{~N}$ ), pressure vessels are used under $200 \quad$ our£oraß@ then redundant water and nitric acid are removed and recycled by heating-condensing method. The luminescent properties of phosphors synthesized with the raw materials produced by this technology equal to or exceed that of the quality phosphors produced by a Japan corporation. The technology can prepare required purity Gallium nitrate with advantages of simple equipment, easy to operate, environments friendly and low cost.
\end{abstract}

\section{Introduction}

Gallium nitrate become one of the important basic materials and the main raw material in the research and production of new high efficiency Cancer treatment drugs [1], thin combustion nox control, fluorescent material, laser material. The demand of Gallium nitrate in the high technology field of new medicine, functional materials and catalysis has been growing as important chemical and pharmaceutical base materials. But the preparation of Gallium nitrate is very difficult and the cost of the mass production of Gallium nitrate increases due to the raw material of Gallium nitrate -- Gallium oxide chemical activity is very stable, not easy to react with nitric acid. Therefore, Gallium nitrate preparation methods, especially the preparation of large quantities of high pure Gallium nitrate method become a research focus.

Few Gallium nitrate preparation methods research work has been reported. Two methods were introduced in "'inorganic compounds synthesized handbook' edited by Japan Chemical society [2]: One is through the repeated evaporation, concentration of the reaction product of metal Gallium or Gallium oxide and concentrated nitric acid, Another method is to cool the Gallium or Gallium hydroxide dissolved nitric acid solution. Yongming Luo [3], Xihui Wang et al. [4] reported the improved method on the basis of the above greatly shorten the production cycle. Because it is very difficult for Gallium oxide to dissolve nitric acid at room temperature, the oxide coated membrane produced by the reaction of Gallium and nitric acid under room temperature prevent further reaction of Gallium with nitric acid, Gallium nitrate crystallization precipitation is hard, and crystallization separation need much time, it makes the whole production cycle is a long time and the cost of production is high. In addition, the use of excessive concentrated nitric acid, unceasingly water washing for the purification and enrichment will produce a large amount of sewage, these also increase the consumption of water and the pollution. The difficult of mass preparation of Gallium nitrate become the bottleneck of application of Gallium nitrate. Shorten the production cycle, reduce production costs, reduce or avoid pollution, reducing the consumption of water becomes Gallium nitrate production, especially mass production Gallium nitrate research key.

In this paper, a novel Gallium nitrate preparation method is reported. The method has the advantages of short production cycle, simple equipments, easy to operate, water saving, energy saving, low cost, little pollution and easy to mass production. By this new method, different pure Gallium nitrate for medicine, laser materials, fluorescent material, and thin combustion nox control 
can be prepared. The successful application of the Gallium nitrate produced by the method in the field of fluorescent material is also illustrated in the paper.

\section{Experiment}

\section{The main raw materials and equipments}

Raw material: Gallium oxide, nitric acid and water. The purity of raw materials is decided according to the request of Gallium nitrate product purity, but must higher than the purity of the product. Experimental apparatus: stainless steel pressure kettle, the type electric heating thermostat (DH-101-3 BS), thermostatic waterbath (HX-100), negative pressure condensing equipment. The negative pressure condensing equipment is homemade. Fig. 1 shows the schematic diagram of negative pressure condensing equipment.

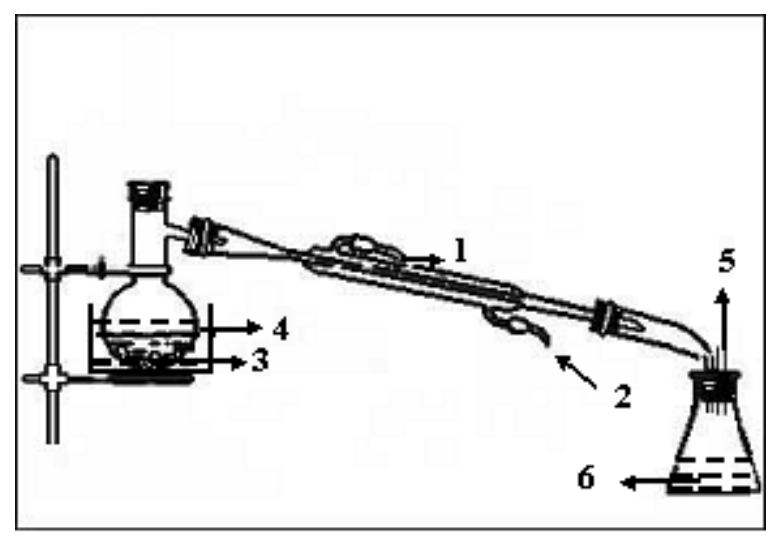

Fig.1 The schematic diagram of negative pressure condensing equipment ( 1 Condensate water outlet, 2 Condensate water import, 3 Gallium nitrate nitric acid solution, 4 Constant temperature water bath, 5 Negative pressure, 6 nitric acid solution )

\section{The preparation process}

The preparation process includes five stages [5]: mixture of raw material stage, high pressure high temperature reaction stage and purification and drying stage. Mixture of law materials: certain purity Gallium oxide, nitrate, water were mixed in stainless steel pressure kettle. The nitric acid was diluted to $5 \sim 16 \mathrm{~N}$ with water. The ratio of Gallium oxide to nitric acid is 1:5 18. High pressure high temperature reaction: the pressure kettle was steeped in $200{ }^{\circ} \mathrm{C}$ water for about 20 hours and rolled over every $15 \sim 20$ minutes. Purification and drying: The excess water and nitric acid in pressure kettle mixture were removed via condensation under $40-80{ }^{\circ} \mathrm{C}$ with the pressure of $0.05 \times 105 \sim 0.05$ $\times 105$ pa. The condensation process should be repeated till the pure of Gallium nitrate reach the requirements.

\section{Results and Discussions}

\section{Advantages of novel preparation method}

Fig. 2 shows the process diagram of novel methods and reported method [1]. Fig. 2 indicates that the novel method has following advantages: The pressure kettle in the novel method accelerated the reaction speed dramatically, thus the process stage time is shortened and production efficiency is improved. Low pressure distillation technology in the novel method makes purification and drying process stage efficiency, water saving and environmental protection. 


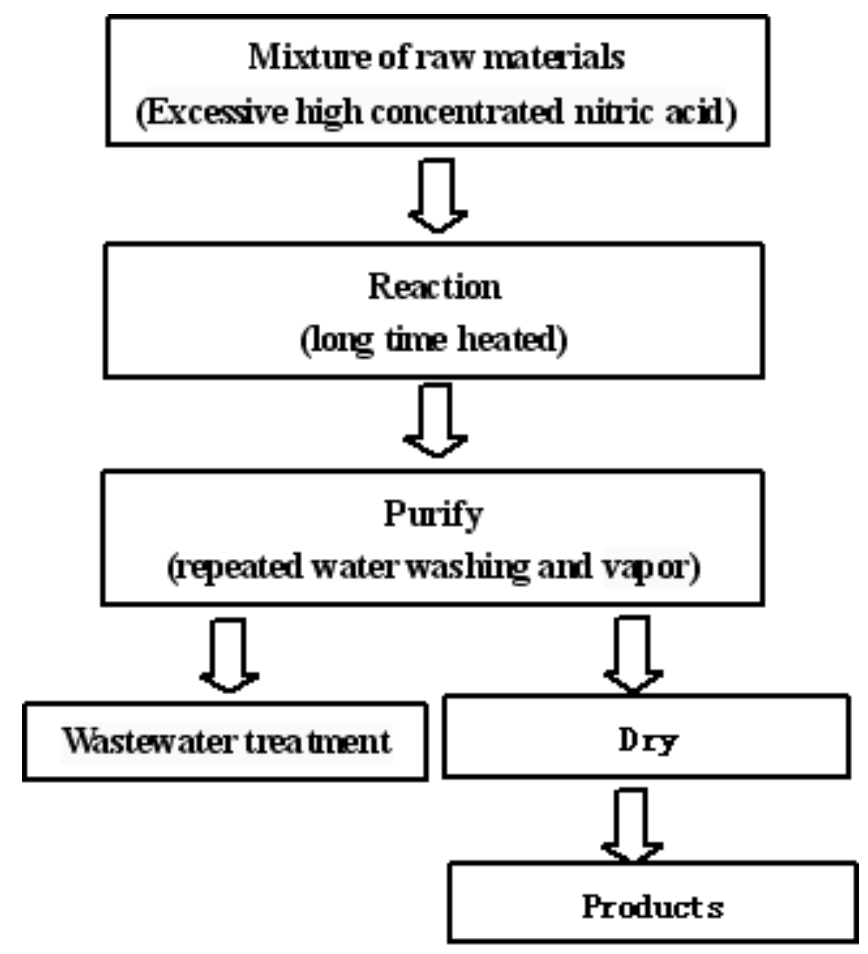

Reported method process dia gram

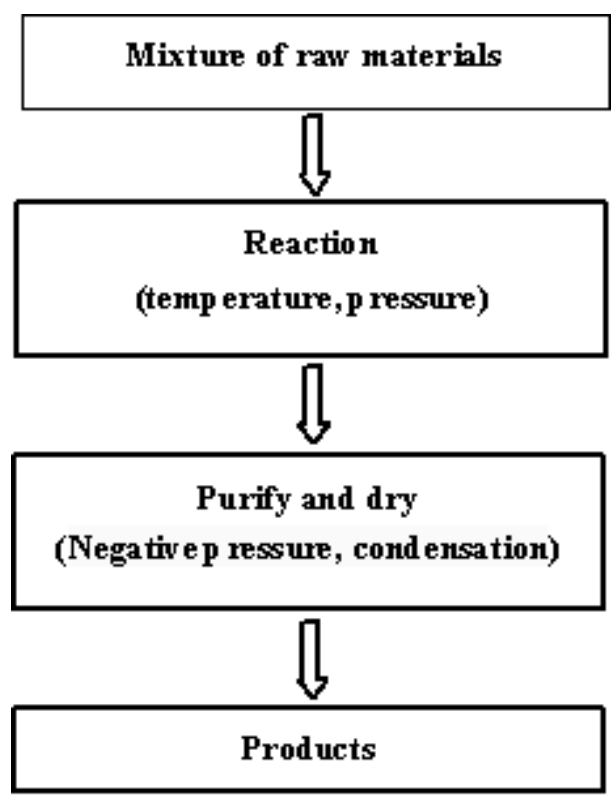

Novel m ethod process diagram

Fig. 2 The process diagram of novel methods and reported method [1]

\section{SEM morphology of prepared Gallium nitrate}

Fig.3 shows SEM image of the prepared Gallium nitrate. The crystals of the prepared Gallium nitrate have the shape of rule rectangular, the size is about $1 \times 3 \mu \mathrm{m}$, the distribution and dispersivity of the particles is rather good. 


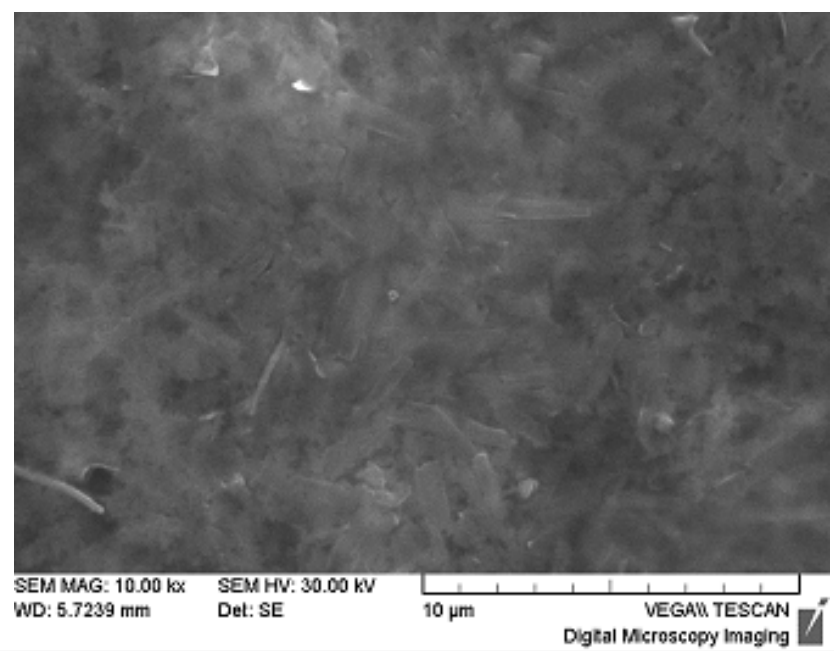

Fig.3 The SEM image of the prepared Gallium nitrate

\section{Application case: prepared Gallium nitrate applied in fluorescent materials}

Phosphors Y3(AlGa)5O12:Tb were synthesized by combustion melting salt aid sol-gel method with high purity terbium nitrate, yttrium nitrate and prepared Gallium nitrate as raw material[4]. The Gallium nitrate was prepared by the novel technology with high purity Gallium oxide (99.95\%), high purity nitric acid (excellent level of pure), deionized water as raw materials. Fig.4 shows the cathodoluminescent properties of phosphors $\mathrm{Y3}(\mathrm{AlGa}) 5 \mathrm{O} 12$ :Tb synthesized using novel method prepared Gallium nitrate and same phosphors from a Japanese company.

Fig.4 indicates that the cathodoluminescent intensity of prepared phosphor is significantly higher than that of Japanese sample: the intensity is about $160 \%$ of the Japanese samples under $0.8 \mathrm{Kv}$ voltage, $120 \%$ of the Japanese sample under $1.0 \mathrm{Kv}$ voltage. The prepared Gallium nitrate can completely satisfy the requirement in the field of the fluorescence.

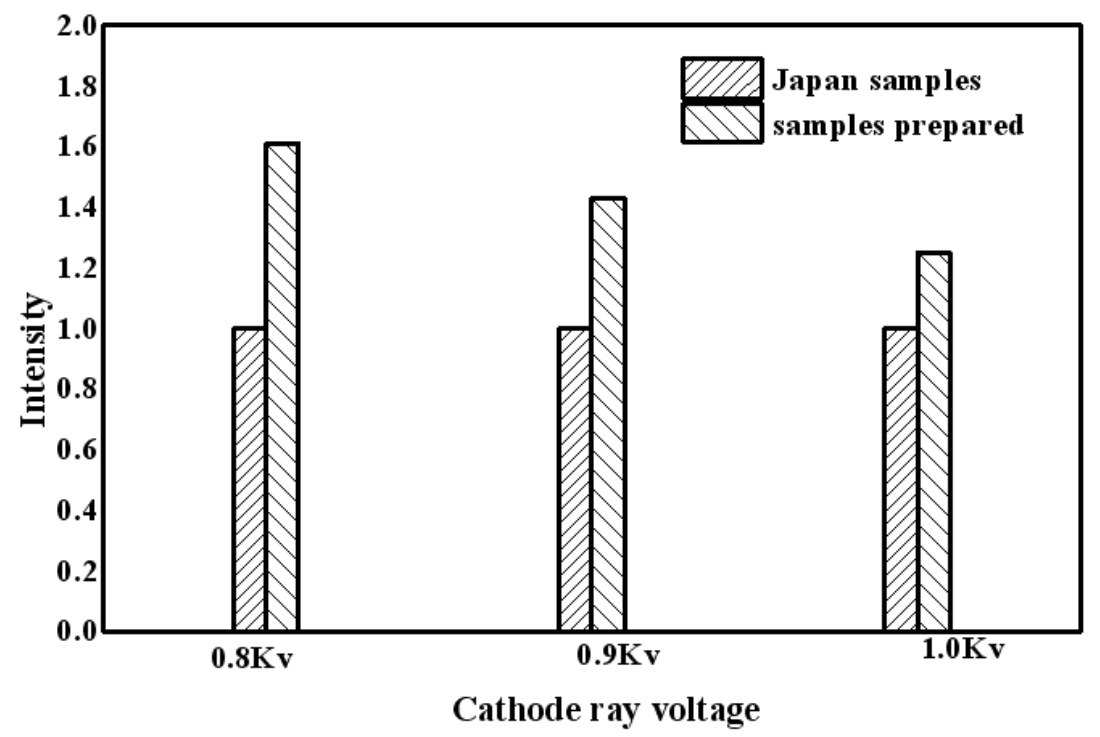

Fig.4 The cathodoluminescent properties of phosphors $\mathrm{Y}_{3}(\mathrm{AlGa})_{5} \mathrm{O}_{12}: \mathrm{Tb}$

\section{Summary}

Adopt low pressure condensation process, the novel method can prepared different purity Gallium nitrate using Gallium oxide, nitric acid and water as raw materials. The novel method includes the following steps: raw materials mixing, high pressure high temperature reaction, purification and drying by low pressure condensation process. The preparation technology can greatly improve the production efficiency, realize the mass production, and achieve low cost, saving water, pollution-free 
green production requirements. The application of the prepared Gallium nitrate in the Y3(AlGa)5O12:Tb phosphors indicates that the Gallium nitrate prepared by the novel method can completely satisfy the requirement in the field of the fluorescence.

\section{Acknowledgements}

This work was financially supported by the National Natural Science Founds of China (61204003), the Jiangxi Natural Science Foundation (20133BAB20005,), Jiangxi Provincial Department of Education (GJJ12270), the Floor Plan of Science and Technology for Higher School of Jiangxi Province (KJLD13034), and the Excellent Young Academic Talent Program of Jiangxi University of Finance and Economics, respectively.

\section{References}

[1] Information on http://baike.so.com/doc/6741080.html

[2] Jiaju An, Zhichun Chen, in: inorganic compounds synthesized handbook (In Chin), edited by Japan Chemical society, Chemical industry press, Beijing(1986), 207.

[3] Yongming Luo, Jiming Hao, Lixin Fu, P.R.China, Patent 1178861C. (2004)

[4] Xihui Wang, Zijian Lü, Gang Wu, P.R.China, Patent 1762825A.(2006)

[5] Quanmao Yü, P.R.China, Patent 200610018594.4.(2006) 